\title{
Flow Stability Analysis of the Shallow Water Equations Model
}

\section{Ashraf S. Aboudi}

College of Computer Science and Mathematics

University of Mosul, Iraq

\section{Received on: 30/11/2008}

\section{ABSTRACT}

Accepted on: 17/03/2009

This paper is devoted to analyze the stability of shallow water of a system of equations that was exposed to disturbancing. This analysis is done by finding the eigenvalues of the system which enables us to investigate the grow of disturbance after setting shallow water equations in linearization form. It is obtain from the results analysis that the equations are stable when the real part of wave velocity is negative ,and unstable when it is positive.

Keywords: flow, stability analysis, shallow water equations model.

$$
\begin{aligned}
& \text { تحليل استقرارية الجريان لنموذج معادلات المياه الضحلة } \\
& \text { أشرف سمعان عبودي } \\
& \text { كلية علوم الحاسبات واليراضيات، جامعة الموصل علودي } \\
& \text { تاريخ قبول البحث:2009/3/17 } \\
& \text { تاريخ استلام البحث:2008/11/30 } \\
& \text { الملخص } \\
& \text { هذا البحث مكرس لتحليل الاستقرارية لمنظومة من معادلات المياه الضحلة التي تعرضت } \\
& \text { لاضطراب، وهذا التحليل تم عن طريق إيجاد القيم الذاتية ( Eigenvalues ) للمنظومة التي تمكننا } \\
& \text { من إيجاد نمو الاضطراب من عدمه وذلك بعد جعل معادلات المياه الضحلة خطية } \\
& \text { (Linearization) } \\
& \text { الجزء الحقيقي لسرعة الموجة كمية سالبة ، ويكون في حالة عدم استقرار عندما تكون هذه الكمية لهادية } \\
& \text { موجبة. } \\
& \text { الكلمات المفتاحية: الجريان، تحليل الاستقرارية، نموذج معادلات المياه الضحلة. } \\
& \text { 1- المقدمة: }
\end{aligned}
$$


وظواهرها، وفي الأرصاد الجوية(Meteorology)، وفي مجال الهندسة والعديد من مسائل العلوم التطبيقية الأخرى ) [6].

إن أي نظام ،ومنه نظام معادلات المياه الضحلة ( Shallow water equations )

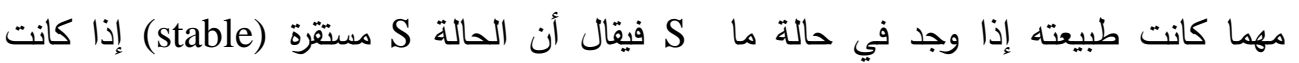
الإزعاجات أو التأثيرات الخارجية التي يتعرض لها النظام لا تؤثر في الحالة S . وكذلك النظام الثمسي على سبيل المثال موجود حالياّ في حالة معتمدة على الزمن ذلك أن الكواكب تدور حول حول

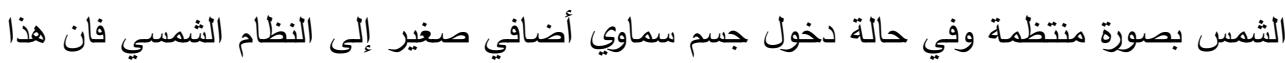

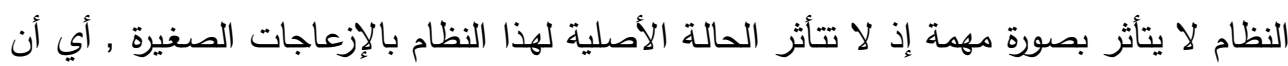

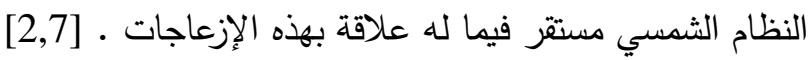
ومن الأمثلة المهمة الأخرى على مفهوم الاستقرارية ( Stability) حركة الأمبيا تحت تأثير

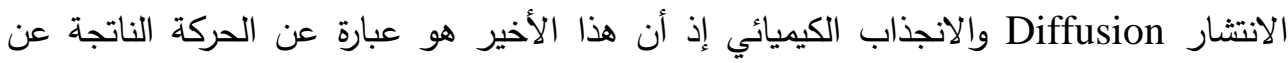

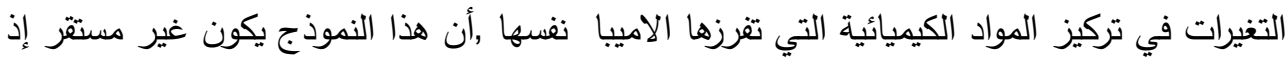

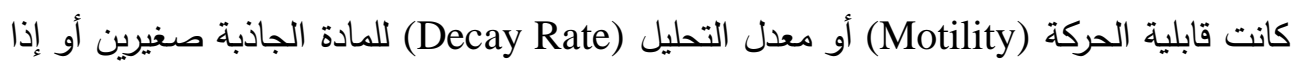
كان معدل الإفراز ( Secretion Rate) أو قوة الانجذاب الكيميائي كبيرين .ولهذه الظاهرة أهمية كبيرة في علم الأحياء التطويري ,والمفهوم نفسه ينطبق على جميع الظواهر الطبيعية الآخرى .

لقد كان اول من درس فكرة الآستقرارية هو العالم الفرنسي الكبير بوانكاريه (poincare) (1854-1912) والذي يُعد من كبار العلماء في العالم. ويقترن موضوع الاستقرار كذلك بأسم العالم

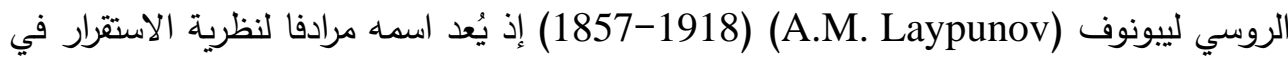
العالم الغربي منذ عام 1960 وان أفكاره وجدت طريقها في الحقول المليئة بالأفكار الخصبة والمثمرة

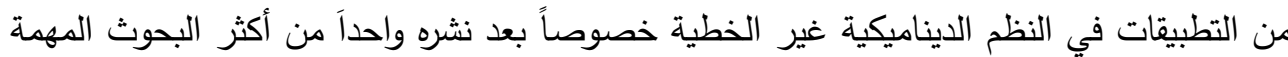
On the General ) في عام 1892 وهذه هي المسألة العامة لاستقرارية الحركة فئسية .[10] (Problem of the stability of Motion

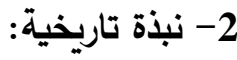

درس كل من العالمين William F.Spotz و Mark A. Taylor عام 1999 طرائق

سريعة لحساب المشتقات الخاصة للدوال الكروية الثكل وذلك لاستخدامها في تقريبات Explicit time - stepping Approximation لقد استخدم الترشيح ( Filtering) لتحليل الاستقرارية لهذه الطريقة والاهتمام قد انصب على 
استخدام طرائق الفورير والترشيح التوافقي الكروي ، وتبين أن نتيجة الطيف تتحقق تقريبا في ثلث

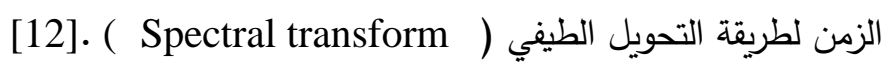
كذلك برهن العالم (Yong Zhou) ) عام 2000 ان سرعة انتشار معادلات المياه

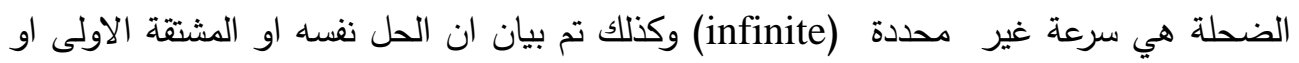

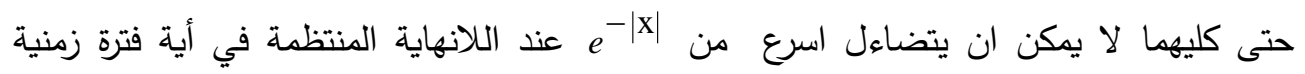

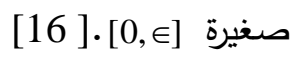

كذلك ناقش كل من العالمين Tomas Chacon و Antonio Dominguez عام 2004 نوعاً من الاثكال العددية المتوازنة من الرتبة الثانية لحل انظمة القطع الزائدة غير المتجانسة

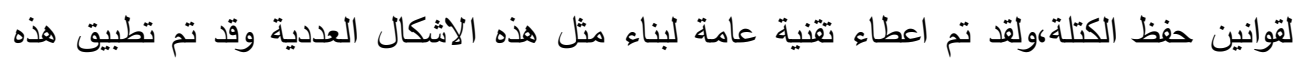

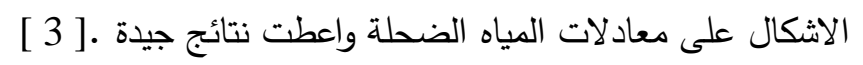
لقد وجد العالمان Fernandez - Feria و Bohorquez عام 2006 الفروقات النوعية بالنسبة الى الاستقرارية المتوازية الخاصة لجريان المياه الضحلة عبر مستوي مائل بميل

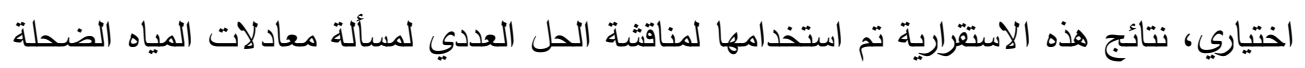

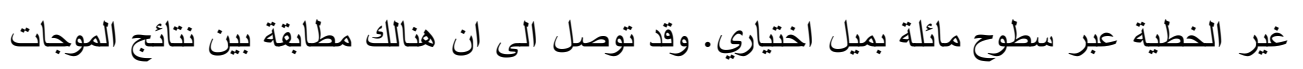

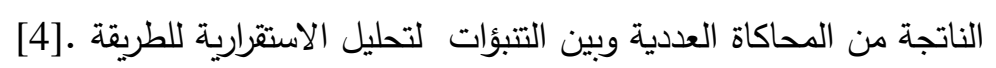
كذلك درس كل من العالمين Luis P.Thomas و و Carlos C.Pena عام 2006 الحلول البسيطة ( smoth) لمعادلات المياه الضحلة، فقد وجدا ان المجموعة الكاملة للحلول نستطيع

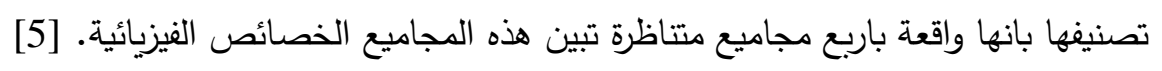

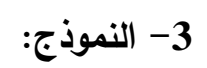

ليكن لدينا مقطع للمياه الضحلة ببعدين كنرمز لمركبة السرعة للمحورين السيني والصادي

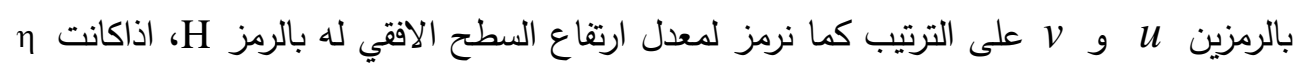

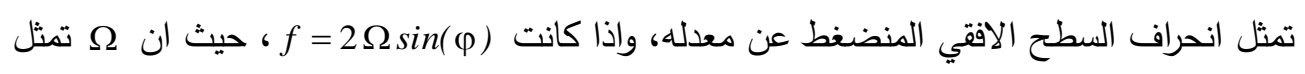

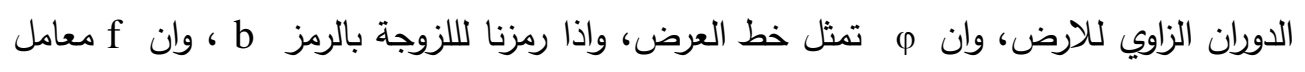

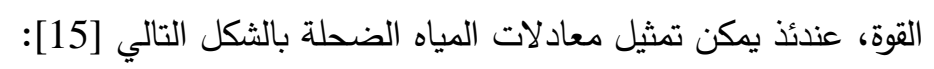

$$
\begin{aligned}
\frac{D u}{\partial t}-\mathrm{fv} & =-\mathrm{g} \frac{\partial \eta}{\partial \mathrm{x}}-b u \\
\frac{D v}{\partial t}+\mathrm{fu} & =-\mathrm{g} \frac{\partial \eta}{\partial \mathrm{y}}-b v \\
\frac{\partial \eta}{\partial \mathrm{t}} & =\frac{\partial(\mathrm{u}(\mathrm{H}+\eta))}{\partial \mathrm{x}}+\frac{\partial(\mathrm{v}(\mathrm{H}+\eta))}{\partial \mathrm{y}}
\end{aligned}
$$




$$
\begin{aligned}
& \frac{D}{D \mathrm{t}}=\frac{\partial}{\partial \mathrm{t}}+u \frac{\partial}{\partial x}+v \frac{\partial}{\partial y} \\
& \frac{\partial \mathrm{u}}{\partial \mathrm{t}}+\mathrm{u} \frac{\partial \mathrm{u}}{\partial \mathrm{x}}+\mathrm{v} \frac{\partial u}{\partial \mathrm{y}}-\mathrm{fv}=-\mathrm{g} \frac{\partial \eta}{\partial \mathrm{x}}-b u \\
& \frac{\partial v}{\partial \mathrm{t}}+\mathrm{u} \frac{\partial \mathrm{v}}{\partial \mathrm{x}}+\mathrm{v} \frac{\partial v}{\partial \mathrm{y}}+\mathrm{fu}=-\mathrm{g} \frac{\partial \eta}{\partial \mathrm{y}}-b v \\
& \frac{\partial \eta}{\partial \mathrm{t}}=\frac{\partial u}{\partial \mathrm{x}}+\frac{\partial v}{\partial \mathrm{y}}
\end{aligned}
$$$$
\text { فان المعادلة (1) تصبح بالثكل : }
$$

$$
u=u(H+\eta), \mathrm{v}=\mathrm{v}(\mathrm{H}+\eta) \quad \text { حيث }
$$

4- المعاملات والمعادلات اللابعدية: (Non Dimensional form ) لغرض ايجاد المعادلات اللابعدية، سوف نعرف بعض القيم اللابعدية وعلى افتراض ان

$$
\begin{aligned}
& \text { un } \\
& x=L x^{*} \quad \mathrm{y}=\mathrm{Ly}^{*} \quad \mathrm{t}=\frac{\mathrm{Lt}^{*}}{\mathrm{u}_{0}} \quad \mathrm{v}=\mathrm{u}_{0} v^{*} \quad \mathrm{u}=\mathrm{u}_{0} u^{*} \quad \eta=\eta_{0} \eta^{*}
\end{aligned}
$$$$
\text { وبتعويض الكميات والقيم اللابعدية في (2) نحصل على المعادلات اللابعدية لمعادلات المياه }
$$

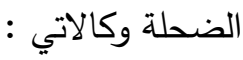

$$
\begin{gathered}
\frac{\partial u^{*}}{\partial \mathrm{t}^{*}}+\mathrm{u}^{*} \frac{\partial \mathrm{u}^{*}}{\partial \mathrm{x}^{*}}+\mathrm{v} * \frac{\partial u^{*}}{\partial \mathrm{y}^{*}}-\frac{L}{u_{0}} \mathrm{fv}^{*}=-\frac{\mathrm{g} \eta_{0}}{\mathrm{u}_{0}^{2}} \frac{\partial \eta^{*}}{\partial \mathrm{x}^{*}}-\frac{L}{u_{0}} b u^{*} \\
\frac{\partial v^{*}}{\partial \mathrm{t}^{*}}+\mathrm{u}^{*} \frac{\partial v^{*}}{\partial \mathrm{x}^{*}}+\mathrm{v}^{*} \frac{\partial v^{*}}{\partial \mathrm{y}^{*}}+\frac{L}{u_{0}} \mathrm{fu}^{*}=-\frac{\mathrm{g \eta}_{0}}{\mathrm{u}_{0}^{2}} \frac{\partial \eta^{*}}{\partial y^{*}}-\frac{L}{u_{0}} b v^{*} \\
\frac{\partial \eta^{*}}{\partial \mathrm{t}^{*}}=\frac{1}{\eta_{0}}\left(\frac{\partial u^{*}}{\partial \mathrm{x}^{*}}+\frac{\partial v^{*}}{\partial \mathrm{y}^{*}}\right)
\end{gathered}
$$

5- تحليل الاستقرارية: (Stability Analysis)

لغرض تحليل الاستقرارية لنموذج معادلات المياه الضحلة المعرفة بـ (3) نجزأ كلا من باستخدام المعادلتين التاليتين $U^{*}, V^{*}$

$$
\begin{aligned}
& u^{*}(x, y, t)=\mathrm{u}_{1}^{*}(x, y)+u_{2}^{*}(x, y, t) \\
& v^{*}(x, y, t)=\mathrm{v}_{1}^{*}(x, y)+v_{2}^{*}(x, y, t)
\end{aligned}
$$


حيث ان

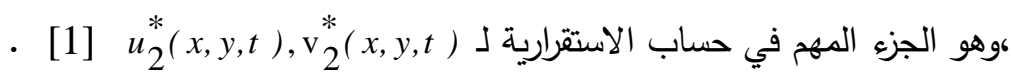

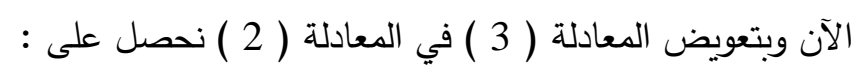
$\frac{\partial\left(\mathrm{u}_{1}^{*}+u_{2}^{*}\right)}{\partial \mathrm{t}^{*}}+\left(\mathrm{u}_{1}^{*}+u_{2}^{*}\right) \frac{\partial\left(\mathrm{u}_{1}^{*}+u_{2}^{*}\right)}{\partial \mathrm{x}^{*}}+\left(v_{1}^{*}+v_{2}^{*}\right.$
$=-\left(g_{1}+g_{2}\right) \frac{n_{0}}{u_{0}^{2}} \frac{\partial\left(\eta_{1}^{*}+\eta_{2}^{*}\right)}{\partial \mathrm{x}^{*}}-b \frac{L}{u_{0}}\left(u_{1}^{*}+u_{2}^{*}\right)$

$\frac{\partial\left(v_{1}^{*}+v_{2}^{*}\right)}{\partial \mathrm{t}^{*}}+\left(\mathrm{u}_{1}^{*}+u_{2}^{*}\right) \frac{\partial\left(\mathrm{v}_{1}^{*}+v_{2}^{*}\right)}{\partial \mathrm{x}^{*}}+\left(v_{1}^{*}+v_{2}^{*}\right) \frac{\partial\left(\mathrm{v}_{1}^{*}+v_{2}^{*}\right)}{\partial \mathrm{y}^{*}}-\frac{L}{u_{0}} f\left(u_{1}^{*}+u_{2}^{*}\right)$
$=-\left(g_{1}+g_{2}\right) \frac{\partial\left(\eta_{1}^{*}+\eta_{2}^{*}\right)}{\partial \mathrm{y}^{*}}-b\left(\left(v_{1}^{*}+v_{2}^{*}\right)\right.$

$\frac{\partial\left(v_{1}^{*}+v_{2}^{*}\right)}{\partial \mathrm{t}^{*}}+\left(\mathrm{u}_{1}^{*}+u_{2}^{*}\right) \frac{\partial\left(\mathrm{v}_{1}^{*}+v_{2}^{*}\right)}{\partial \mathrm{x}^{*}}+\left(v_{1}^{*}+v_{2}^{*}\right) \frac{\partial\left(\mathrm{v}_{1}^{*}+v_{2}^{*}\right)}{\partial \mathrm{y}^{*}}-\frac{L}{u_{0}} f\left(u_{1}^{*}+u_{2}^{*}\right)$
$=-\left(g_{1}+g_{2}\right) \frac{\partial\left(\eta_{1}^{*}+\eta_{2}^{*}\right)}{\partial \mathrm{y}^{*}}-b\left(\left(v_{1}^{*}+v_{2}^{*}\right)\right.$

$\frac{\partial\left(\eta_{1}^{*}+\eta_{2}^{*}\right)}{\partial \mathrm{t}^{*}}=\frac{1}{\eta_{0}}\left(\frac{\partial\left(\mathrm{u}_{1}^{*}+u_{2}^{*}\right)}{\partial \mathrm{x}^{*}}+\frac{\partial\left(v_{1}^{*}+v_{2}^{*}\right)}{\partial \mathrm{y}^{*}}\right)$ وبتبسيط المعادلات (4)،(5)،(6) نحصل على :

$\frac{\partial u_{1}^{*}}{\partial \mathrm{t}^{*}}+\frac{\partial u_{2}^{*}}{\partial \mathrm{t}^{*}}+u_{1}^{*} \frac{\partial u_{1}^{*}}{\partial \mathrm{x}^{*}}+u_{2}^{*} \frac{\partial u_{2}^{*}}{\partial \mathrm{x}^{*}}+v_{1}^{*} \frac{\partial u_{1}^{*}}{\partial \mathrm{y}^{*}}+v_{2}^{*} \frac{\partial \mathrm{u}_{2}^{*}}{\partial \mathrm{y}^{*}}-f v_{1}^{*}-f v_{2}^{*}$

$=-g_{1} \frac{\partial \eta_{1}^{*}}{\partial x^{*}}-g_{2} \frac{\partial \eta_{2}^{*}}{\partial x^{*}}-b u_{1}^{*}-b u_{2}^{*}$

$$
\begin{aligned}
& \frac{\partial v_{1}^{*}}{\partial \mathrm{t}^{*}}+\frac{\partial v_{2}^{*}}{\partial \mathrm{t}^{*}}+u_{1}^{*} \frac{\partial v_{1}^{*}}{\partial \mathrm{x}^{*}}+u_{2}^{*} \frac{\partial v_{2}^{*}}{\partial \mathrm{x}^{*}}+v_{1}^{*} \frac{\partial v_{1}^{*}}{\partial \mathrm{y}^{*}}+v_{2}^{*} \frac{\partial v_{2}^{*}}{\partial \mathrm{y}^{*}}+f u_{1}^{*}+f u_{2}^{*} \\
& =-g_{1} \frac{\partial \eta_{1}^{*}}{\partial y^{*}}-g_{2} \frac{\partial \eta_{2}^{*}}{\partial y^{*}}-b v_{1}^{*}-b v_{2}^{*} \\
& \frac{\partial \eta_{1}^{*}}{\partial \mathrm{t}^{*}}+\frac{\partial \eta_{2}^{*}}{\partial \mathrm{t}^{*}}=\frac{\partial u_{1}^{*}}{\partial \mathrm{x}^{*}}+\frac{\partial u_{2}^{*}}{\partial \mathrm{x}^{*}}+\frac{\partial v_{1}^{*}}{\partial \mathrm{y}^{*}}+\frac{\partial v_{2}^{*}}{\partial \mathrm{y}^{*}}
\end{aligned}
$$


وبتجزئة المعادلات (7) ، (8) ، (9) الى حالتي الاستقرار والاضطراب نحصل على :

$\frac{\partial u_{1}^{*}}{\partial \mathrm{t}^{*}}+u_{1}^{*} \frac{\partial u_{1}^{*}}{\partial \mathrm{x}^{*}}+v_{1}^{*} \frac{\partial u_{1}^{*}}{\partial \mathrm{y}^{*}}-f v_{1}^{*}=-g_{1} \frac{\partial \eta_{1}^{*}}{\partial x^{*}}-b u_{1}^{*}$

$\frac{\partial u_{2}^{*}}{\partial \mathrm{t}^{*}}+u_{2}^{*} \frac{\partial u_{2}^{*}}{\partial \mathrm{x}^{*}}+v_{2}^{*} \frac{\partial u_{2}^{*}}{\partial \mathrm{y}^{*}}-f v_{2}^{*}=-g_{2} \frac{\partial \eta_{2}^{*}}{\partial x^{*}}-b u_{2}^{*}$

$\frac{\partial v_{1}^{*}}{\partial \mathrm{t}^{*}}+u_{1}^{*} \frac{\partial v_{1}^{*}}{\partial \mathrm{x}^{*}}+v_{1}^{*} \frac{\partial v_{1}^{*}}{\partial \mathrm{y}^{*}}+f u_{1}^{*}=-g_{1} \frac{\partial \eta_{1}^{*}}{\partial y^{*}}-b v_{1}^{*}$

$\frac{\partial v_{2}^{*}}{\partial \mathrm{t}^{*}}+u_{2}{ }^{*} \frac{\partial v_{2}^{*}}{\partial \mathrm{x}^{*}}+v_{2}^{*} \frac{\partial v_{2}^{*}}{\partial \mathrm{y}^{*}}+f u_{2}^{*}=-g_{2} \frac{\partial \eta_{2}^{*}}{\partial y^{*}}-b v_{2}^{*}$

$\frac{\partial \eta_{1}^{*}}{\partial \mathrm{t}^{*}}=\frac{\partial u_{1}^{*}}{\partial \mathrm{x}^{*}}+\frac{\partial v_{1}^{*}}{\partial \mathrm{y}^{*}}$

$\frac{\partial \eta_{2}^{*}}{\partial \mathrm{t}^{*}}=\frac{\partial u_{2}^{*}}{\partial \mathrm{x}^{*}}+\frac{\partial v_{2}^{*}}{\partial \mathrm{y}^{*}}$

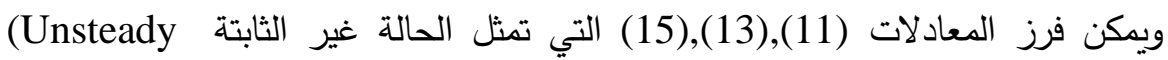

(بكتابتها بالثكل: state)

$\frac{\partial u_{2}^{*}}{\partial t^{*}}+u_{2}^{*} \frac{\partial u_{2}^{*}}{\partial x^{*}}+v_{2}^{*} \frac{\partial u_{2}^{*}}{\partial y^{*}}-f v_{2}^{*}=-g_{2} \frac{\partial \eta_{2}^{*}}{\partial x^{*}}-b u_{2}^{*}$

$\frac{\partial v_{2}^{*}}{\partial t^{*}}+u_{2}^{*} \frac{\partial v_{2}^{*}}{\partial x^{*}}+v_{2}^{*} \frac{\partial v_{2}^{*}}{\partial y^{*}}-f u_{2}^{*}=-g_{2} \frac{\partial \eta_{2}^{*}}{\partial y^{*}}-b v_{2}^{*}$

$\frac{\partial \eta_{2}^{*}}{\partial t^{*}}=\frac{\partial u_{2}^{*}}{\partial x^{*}}+\frac{\partial v_{2}^{*}}{\partial y^{*}}$ 


\section{6- الاضطراب الحادث بالاتجاهين X, X}

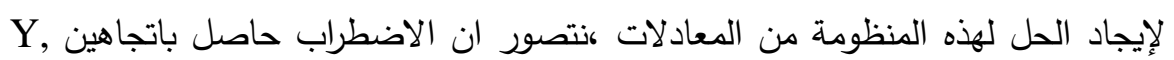

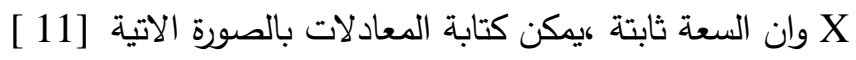

$$
\begin{aligned}
& u_{2}^{*}=A_{1} e^{\alpha \mathrm{t}+\mathrm{i}\left(\mathrm{k}_{1} \mathrm{x}+\mathrm{k}_{2} \mathrm{y}\right)} \\
& v_{2}^{*}=A_{2} e^{\alpha \mathrm{t}+\mathrm{i}\left(\mathrm{k}_{1} \mathrm{x}+\mathrm{k}_{2} \mathrm{y}\right)} \\
& \eta_{2}^{*}=A_{3} e^{\alpha \mathrm{t}+\mathrm{i}\left(\mathrm{k}_{1} \mathrm{x}+\mathrm{k}_{2} \mathrm{y}\right)}
\end{aligned}
$$

حيث أن K $K_{1}, K_{2}$ قيم حقيقية لابعدية لطول الموجة بالاتجاه Y, X على التوالي ، $\alpha$ هي سرعة

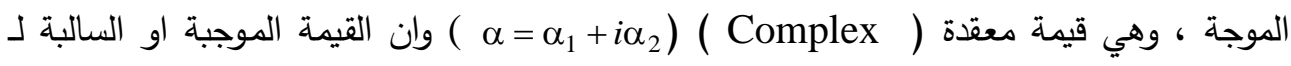

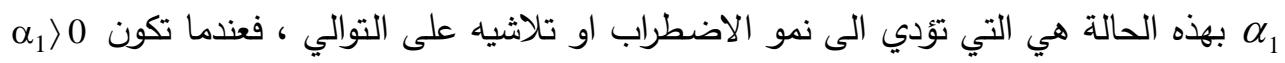

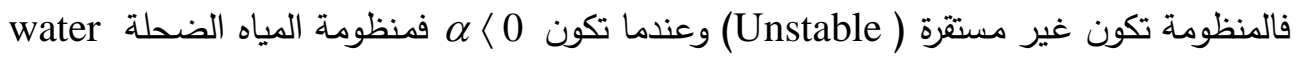
( تكون مستقرة (Shallow equations ) سعة الموجة ( ) (Amplitude) (مدال

بعد إهمال الحدود الصغيرة وغير الخطية بالنسبة الى الاضطراب، يمكن كتابة منظومة المعادلات

$$
\begin{aligned}
& \frac{\partial u_{2}^{*}}{\partial t^{*}}-\frac{L}{u_{0}} f v_{2}^{*}=-\frac{g_{2} \eta_{0}}{u_{0}^{2}} \frac{\partial \eta_{2}^{*}}{\partial x^{*}}-\frac{L}{u_{0}} b u_{2}^{*} \\
& \frac{\partial v_{2}^{*}}{\partial t^{*}}-\frac{L}{u_{0}} f u_{2}^{*}=-\frac{g_{2} \eta_{0}}{u_{0}^{2}} \frac{\partial \eta_{2}^{*}}{\partial y^{*}}-\frac{L}{u_{0}} b v_{2}^{*} \\
& \frac{\partial \eta_{2}^{*}}{\partial t^{*}}=\frac{1}{\eta_{0}}\left(\frac{\partial u_{2}^{*}}{\partial x^{*}}+\frac{\partial v_{2}^{*}}{\partial y^{*}}\right)
\end{aligned}
$$

$$
\text { وبتعويض (17) في (18) نحصل على ما يأتي : }
$$




$$
\begin{aligned}
& \frac{\partial u_{2}^{*}}{\partial t}-\frac{L}{u_{0}} f v_{2}^{*}=-\frac{\eta_{0}}{u_{0}^{2}} g_{2} \frac{\partial \eta_{2}^{*}}{\partial x^{*}}-\frac{L}{u_{0}} b u_{2}^{*} \\
& \frac{\partial\left(A_{1} e^{\alpha \mathrm{t}+\mathrm{i}\left(\mathrm{k}_{1} x+k_{2} \mathrm{y}\right)}\right)}{\partial t}-\frac{L}{u_{0}} f\left(A_{2} \mathrm{e}^{\alpha \mathrm{t}+\mathrm{i}\left(\mathrm{k}_{1} \mathrm{x}+\mathrm{k}_{2} \mathrm{y}\right)}\right)=-g_{2} \frac{n_{0}}{u_{0}^{2}} \frac{\partial\left(A_{3} e^{\alpha \mathrm{t}+\mathrm{i}\left(\mathrm{k}_{1} x+\mathrm{k}_{2} y\right.}\right)}{\partial x}-\frac{L}{u_{0}} b\left(A_{1} e^{\alpha \mathrm{t}+\mathrm{i}\left(\mathrm{k}_{1} \mathrm{x}+\mathrm{k}_{2} \mathrm{y}\right)}\right) \\
& \alpha \mathrm{A}_{1} e^{\alpha \mathrm{t}+\mathrm{i}\left(\mathrm{k}_{1} \mathrm{x}+\mathrm{k}_{2} y\right)}-\frac{A_{2} L}{u_{0}} f \mathrm{e}^{\alpha \mathrm{t}+\mathrm{i}\left(\mathrm{k}_{1} \mathrm{x}+\mathrm{k}_{2} \mathrm{y}\right)}=-\frac{g_{2} A_{3} \eta_{0}}{u_{0}^{2}} \mathrm{~K}_{1} \mathrm{ie}^{\alpha \mathrm{t}+\mathrm{i}\left(\mathrm{k}_{1} \mathrm{x}+\mathrm{k}_{2} \mathrm{y}\right)}-\frac{A_{1} L}{u_{0}^{2}} \mathrm{~b} \mathrm{e}^{\alpha \mathrm{t}+\mathrm{i}\left(\mathrm{k}_{1} \mathrm{x}+\mathrm{k}_{2} \mathrm{y}\right)} \\
& \left(\alpha \mathrm{A}_{1}-\frac{A_{2} L}{u_{0}} \mathrm{f}+\frac{g_{2} A_{3} \eta_{0}}{u_{0}^{2}} \mathrm{~K}_{1} \mathrm{i}+\frac{A_{1} L}{u_{0}^{2}} \mathrm{~b}\right) \mathrm{e}^{\alpha \mathrm{t}+\mathrm{i}\left(\mathrm{k}_{1} \mathrm{x}+\mathrm{k}_{2} \mathrm{y}\right)}=0 \\
& \text { وبما أن: } \\
& e^{\alpha \mathrm{t}+\mathrm{i}\left(\mathrm{k}_{1} \mathrm{x}+\mathrm{k}_{2} \mathrm{y}\right)} \neq 0 \\
& \left(\alpha A_{1}-\frac{A_{2} L}{u_{0}} \mathrm{f}+\frac{g_{2} A_{3} \eta_{0}}{u_{0}^{2}} \mathrm{~K}_{1} \mathrm{i}+\frac{A_{1} L}{u_{0}^{2}} \mathrm{~b}\right)=0 \\
& \text { فان: }
\end{aligned}
$$

كذلك فان

$$
\begin{aligned}
& \frac{\partial v_{2}^{*}}{\partial t^{*}}-\frac{L}{u_{0}} f u_{2}^{*}=-\frac{\eta_{0} g_{2}}{u_{0}^{2}} \frac{\partial \eta_{2}^{*}}{\partial y^{*}}-\frac{L}{u_{0}} b v_{2}^{*} \\
& \left(\alpha \mathrm{A}_{2}-\frac{\mathrm{A}_{1} L}{\mathrm{u}_{0}} \mathrm{f}+\frac{\mathrm{g}_{2} \mathrm{i} \mathrm{k}_{2} \mathrm{~A}_{3} \eta_{0}}{\mathrm{u}_{0}^{2}}+\frac{\mathrm{A}_{2} L}{\mathrm{u}_{0}} \mathrm{~b}\right)=0
\end{aligned}
$$

وكذلك

$-\frac{A_{1} K_{1} \mathrm{i}}{\eta_{0}}-\frac{A_{2} K_{2} \mathrm{i}}{\eta_{0}}+\alpha \mathrm{A}_{3}=0$

وبجمع الحدود المتشابهة وترتيب المعادلات( 19) ) ) , (21 ) نحصل على ما يأتي : 


$$
\begin{aligned}
& \left(\alpha u_{0}^{2}+L b\right) \mathrm{A}_{1}-L u_{0} f \mathrm{~A}_{2}+\mathrm{g}_{2} \eta_{0} \mathrm{ik}_{1} \mathrm{~A}_{3}=0 \\
& -L u_{0} f \mathrm{~A}_{1}+\left(\alpha u_{0}^{2}+L u_{0} b\right) \mathrm{A}_{2}+g_{2} \mathrm{k}_{2} \eta_{0} i \mathrm{~A}_{3}=0 \\
& -i \mathrm{k}_{1} A_{1}-i \mathrm{k}_{2} \mathrm{~A}_{2}+\alpha \eta_{0} \mathrm{~A}_{3}=0
\end{aligned}
$$

ومن خلال هذه المعادلة نقوم بإيجاد القيم الذاتية (EigenValues) وذلك بكتابة نظام المعادلات

$$
\begin{aligned}
& {\left[\begin{array}{lll}
s_{1} & s_{2} & s_{3} \\
s_{4} & s_{5} & s_{6} \\
s_{7} & s_{8} & s_{9}
\end{array}\right]=0} \\
& s_{1}=\alpha u_{0}^{2}+L b u_{2} \quad \mathrm{~s}_{2}=-L u_{0} f v_{2} \quad \mathrm{~s}_{3}=\mathrm{g}_{2} \eta_{0} \mathrm{ik}_{1} \quad \mathrm{~s}_{4}=L u_{0} f u_{2} \\
& \mathrm{~s}_{5}=\alpha u_{0}^{2}+L u_{0} b v_{2} \quad \mathrm{~s}_{6}=\mathrm{g}_{2} \eta_{0} \mathrm{ik}_{2} \quad s_{7}=-\mathrm{i} \mathrm{k}_{1} \quad \mathrm{~s}_{8}=-\mathrm{i} \mathrm{k}_{2} \quad \mathrm{~s}_{9}=\alpha \eta_{0} \\
& \operatorname{det}\left(\left[\begin{array}{lll}
S_{1} & S_{2} & S_{3} \\
S_{4} & S_{5} & S_{6} \\
S_{7} & S_{8} & S_{9}
\end{array}\right]-\left[\begin{array}{ccc}
\lambda & 0 & 0 \\
0 & \lambda & 0 \\
0 & 0 & \lambda
\end{array}\right]\right)=0 \\
& \left|\begin{array}{ccc}
\left(\alpha u_{0}+L b u_{2}\right)-\lambda & -L u_{0} f v_{2} & g_{2} \eta_{0} i k_{1} \\
L u_{0} f u_{2} & \left(\alpha u_{0}^{2}+L u_{0} b v_{2}\right)-\lambda & g_{2} \eta_{0} i k_{2} \\
-i k_{1} & -i k_{2} & \alpha \eta_{0}-\lambda
\end{array}\right|=0 \\
& \text { و بايجاد قيمة المحدد : } \\
& {\left[\left(\alpha u_{0}+L b u_{2}\right)-\lambda\right] *\left|\begin{array}{cc}
\left(\alpha u_{0}^{2}+L u_{0} b v_{2}\right)-\lambda & g_{2} \eta_{0} i k_{2} \\
-i k_{2} & \alpha \eta_{0}-\lambda
\end{array}\right|+L u_{0} f v_{2} *\left|\begin{array}{cc}
L u_{0} f u_{2} & g_{2} \eta_{0} i k_{2} \\
-i k_{1} & \alpha \eta_{0}-\lambda
\end{array}\right|} \\
& +g_{2} \eta_{0} i k_{1} *\left|\begin{array}{cc}
L u_{0} f u_{2} & \left(\alpha u_{0}^{2}+L u_{0} b v_{2}\right)-\lambda \\
-i k_{1} & -i k_{2}
\end{array}\right|=0 \\
& {\left[\left(\alpha u_{0}^{2}+L b u_{2}\right)-\lambda\right] *\left[\left(\left(\alpha u_{0}^{2}+L u_{0} b v_{2}\right)-\lambda\right)\left(\alpha \eta_{0}-\lambda\right)+\left(g_{2} \eta_{0} i * i k_{2}^{2}\right)\right]+L u_{0} f v_{2}} \\
& *\left\{\left(\alpha \eta_{0}-\lambda\right) * L u_{0} f u_{2}+i^{2} k_{1} * g_{2} \eta_{0} k_{2}\right\}+g_{2} \eta_{0} i k_{1} *\left\{L u_{0} f u_{2} *\left(-i k_{2}\right)+i k_{1} *\left\{\left(\alpha u_{0}^{2}+L u_{0} b v_{2}\right)-\lambda\right\}\right\}=0
\end{aligned}
$$


$\left[\left(\alpha u_{0}^{2}+L b u_{2}\right)-\lambda\right] *\left[\alpha^{2} u_{0}^{2} \eta_{0}+\alpha \eta_{0} L U_{0} b v_{2}-\alpha \eta_{0} \lambda-\alpha \lambda u_{0}^{2}-\lambda \mathrm{Lu}_{0} b v_{2}+\lambda^{2}-g_{2} \eta_{0} k_{2}^{2}\right]+L u_{0} f v_{2}$

$*\left[L u_{0} f u_{2}\left(\alpha \eta_{0}-\lambda\right)+i^{2} g_{2} k_{1} k_{2} \eta_{0}\right]+g_{2} \eta_{0} i k_{1} *\left[-i k_{2} L u_{0} f u_{2}+i k_{1}\left(\left(\alpha u_{0}^{2}+L u_{0} b v_{2}\right)-\lambda\right]=0\right.$

$\left[\left(\alpha u_{0}^{2}+L b u_{2}\right)-\lambda\right] *\left[\alpha^{2} u_{0}^{2} \eta_{0}+\alpha \eta_{0} L u_{0} b v_{2}-\alpha \eta_{0} \lambda-\alpha \lambda u_{0}^{2}-\lambda L u_{0} b v_{2}+\lambda^{2}\right]+L u_{0} f v_{2}\left[L u_{0} f u_{2} \alpha \eta_{0}\right.$

$-L u_{0} f u_{2} \lambda+i^{2} k_{1} k_{2} g_{2} \eta_{0} J+g_{2} \eta_{0} i k_{1}\left[-L u_{0} f u_{2} i k_{2}+\alpha u_{0}^{2} i k_{1}+L u_{0} b v_{2} i k_{1}-i k_{1} \lambda\right]=0$

\section{وبفتح الأقواس وإعادة ترتيب الحدود نجد أن :}

$\alpha^{3} u_{0}^{4} \eta_{0}+L b u_{2} \alpha^{2} u_{0}^{2} \eta_{0}-\alpha^{2} u_{0}^{2} \eta_{0} \lambda+\alpha^{2} u_{0}^{3} \eta_{0} L b v_{2}+L^{2} b u_{2} b v_{2} \alpha \eta_{0} u_{0}-\lambda \alpha \eta_{0} L u_{0} b v_{2}$

$-\alpha^{2} u_{0}^{2} \eta_{0} \lambda-L b u_{2} \alpha \eta_{0} \lambda+\alpha \eta_{0} \lambda^{2}-\alpha^{2} u_{0}^{4} \lambda-L b u_{2} \alpha \lambda u_{0}^{2}+\lambda^{2} \alpha u_{0}^{2}-\alpha u_{0}^{3} L b v_{2} \lambda-L^{2} b u_{2} u_{0} b v_{2} \lambda$

$+\lambda^{2} L u_{0} b v_{2}+\alpha u_{0}^{2} \lambda^{2}+L b u_{2} \lambda^{2}-\lambda^{3}+L^{2} u_{0}^{2} f v_{2} f u_{2} \alpha \eta_{0}-L^{2} u_{0}^{2} f v_{2} f_{u_{2}} \lambda+L u_{0} f v_{2} i^{2} k_{1} k_{2} g_{2} \eta_{0}$

$-g_{2} \eta_{0} i^{2} k_{1} L u_{0} f u_{2} k_{2}+\alpha u_{0}^{2} i^{2} k_{1}^{2} g_{2} \eta_{0}+g_{2} \eta_{0} i^{2} k_{1}^{2} L U_{0} b v_{2}-i^{2} k_{1} \lambda g_{2} \eta_{0} k_{1}=0$

$$
\text { وبتجميع الحدود المتثابهة والضرب ×(-1) نحصل على ما يأتي : }
$$

$-\lambda^{3}+\left(\alpha \eta_{0}+\alpha u_{0}^{2}+L u_{0} b v_{2}+\alpha u_{0}^{2}+L b u_{2}\right) * \lambda^{2}+\left(-\alpha^{2} u_{0}^{2} \eta_{0}-\alpha \eta_{0} L u_{0} b v_{2}-\alpha^{2} u_{0}^{2} \eta_{0}\right.$

$\left.-L b u_{2} \alpha \eta_{0}-\alpha^{2} u_{0}^{4}-L b u_{2} \alpha u_{0}-\alpha u_{0}^{3} L b v_{2}-L^{2} b u_{2} u_{0} b v_{2}-L^{2} u_{0}^{2} f v_{2} f u_{2}-i^{2} k_{1}^{2} g_{2} \eta_{0}\right) * \lambda$

$+\left(\alpha^{3} u_{0}^{4} \eta_{0}+L b u_{2} \alpha^{2} u_{0}^{2} \eta_{0}+\alpha^{2} u_{0}^{3} \eta_{0} L b v_{2}+L^{2} b u_{2} b v_{2} \alpha \eta_{0} u_{0}+L^{2} u_{0}^{2} f v_{2} f u_{2} \alpha \eta_{0}\right.$

$\left.+L u_{0} f v_{2} i^{2} k_{1} k_{2} g_{2} \eta_{0}-g_{2} \eta_{0} i^{2} k_{1} L u_{0} f u_{2} k_{2}+\alpha u_{0}^{2} i^{2} k_{1}^{2} g_{2} \eta_{0}+g_{2} \eta_{0} i^{2} k_{1}^{2} L u_{0} b v_{2}\right)=0$

$\lambda^{3}+\left(-\alpha \eta_{0}-2 \alpha u_{0}^{2}-L u_{0} b v_{2}-L b u_{2}\right) * \lambda^{2}+\left(\alpha^{2} u_{0}^{2} \eta_{0}+\alpha \eta_{0} L u_{0} b v_{2}+\alpha^{2} u_{0}^{2} \eta_{0}+L b u_{2} \alpha \eta_{0}\right.$

$\left.+\alpha^{2} u_{0}^{4}+L b u_{2} \alpha u_{0}+\alpha u_{0}^{3} L b v_{2}+L^{2} b u_{2} u_{0} b v_{2}+L^{2} u_{0}^{2} f v_{2} f u_{2}+i^{2} k_{1}^{2} g_{2} \eta_{0}\right) * \lambda$

$-\left(\alpha^{3} u_{0}^{4} \eta_{0}+L b u_{2} \alpha^{2} u_{0}^{2} \eta_{0}+\alpha^{2} u_{0}^{3} \eta_{0} L b v_{2}+L^{2} b u_{2} b v_{2} \alpha \eta_{0} u_{0}+L^{2} u_{0}^{2} f v_{2} f u_{2} \alpha \eta_{0}\right.$

$\left.+L u_{0} f v_{2} i^{2} k_{1} k_{2} g_{2} \eta_{0}-g_{2} \eta_{0} i^{2} k_{1} L u_{0} f u_{2} k_{2}+\alpha u_{0}^{2} i^{2} k_{1}^{2} g_{2} \eta_{0}+g_{2} \eta_{0} i^{2} k_{1}^{2} L u_{0} b v_{2}\right)=0$

وبالتالي يكون لدينا:

$\lambda^{3}+\xi_{1} \lambda^{2}+\xi_{2} \lambda+\xi_{3}=0$ 


$$
\begin{aligned}
& \xi_{1}=-\alpha \eta_{0}-2 \alpha u_{0}^{2}-L u_{0} b v_{2}-L b u_{2} \\
& \xi_{2}=\alpha^{2} u_{0}^{2} \eta_{0}+\alpha \eta_{0} L u_{0} b v_{2}+\alpha^{2} u_{0}^{2} \eta_{0}+L b u_{2} \alpha \eta_{0}+\alpha^{2} u_{0}^{4}+L b u_{2} \alpha u_{0}+\alpha u_{0}^{3} L b v_{2} \\
& +L^{2} b u_{2} u_{0} b v_{2}+L^{2} u_{0}^{2} f v_{2} f u_{2}+i^{2} k_{1}^{2} g_{2} \eta_{0} \\
& \xi_{3}=-\left(\alpha^{3} u_{0}^{4} \eta_{0}+L b u_{2} \alpha^{2} u_{0}^{2} \eta_{0}+\alpha^{2} u_{0}^{3} \eta_{0} L b v_{2}+L^{2} b u_{2} b v_{2} \alpha \eta_{0} u_{0}+L^{2} u_{0}^{2} f v_{2} f u_{2} \alpha \eta_{0}\right. \\
& \left.+L u_{0} f v_{2} i^{2} k_{1} k_{2} g_{2} \eta_{0}-g_{2} \eta_{0} i^{2} k_{1} L u_{0} f u_{2} k_{2}+\alpha u_{0}^{2} i^{2} k_{1}^{2} g_{2} \eta_{0}+g_{2} \eta_{0} i^{2} k_{1}^{2} L u_{0} b v_{2}\right) \\
& \text { وبعد حل متعددة الحدود (23) برمجيا باستخدام نظام (13) (13] تم ايجاد جذورا لمعادلة } \\
& \text { كما يأتي P, } \mathrm{P}, \pm \mathrm{q} \\
& P=\frac{1}{6}\left[36 \xi_{2} \xi_{1}-108 \xi_{3}-8 \xi_{1}^{3}+12 \sqrt{12 \xi_{2}^{3}-3 \xi_{2}^{2} \xi_{1}^{2}-54 \xi_{2} \xi_{1} \xi_{3}+81 \xi_{3}^{2}+12 \xi_{3} \xi_{1}^{3}}\right]^{(1 / 3)} \\
& -\frac{6\left(\frac{1}{3} \xi_{2}-\frac{1}{9} \xi_{1}^{2}\right)}{\left[36 \xi_{2} \xi_{1}-108 \xi_{3}-8 \xi_{1}^{3}+12 \sqrt{12 \xi_{2}^{3}-3 \xi_{2}^{2} \xi_{1}^{2}-54 \xi_{2} \xi_{1} \xi_{3}+81 \xi_{3}^{2}+12 \xi_{3} \xi_{1}^{3}}\right]^{(1 / 3)}}-\frac{1}{3} \xi_{1} \\
& q_{1}=-\frac{1}{12}\left[36 \xi_{2} \xi_{1}-108 \xi_{3}-8 \xi_{1}^{3}+12 \sqrt{12 \xi_{2}^{3}-3 \xi_{2}^{2} \xi_{1}^{2}-54 \xi_{2} \xi_{1} \xi_{3}+81 \xi_{3}^{2}+12 \xi_{3} \xi_{1}^{3}}\right]^{(1 / 3)} \\
& +\frac{3\left(\frac{1}{3} \xi_{2}-\frac{1}{9} \xi_{1}^{2}\right)}{\left[36 \xi_{2} \xi_{1}-108 \xi_{3}-8 \xi_{1}^{3}+12 \sqrt{12 \xi_{2}^{3}-3 \xi_{2}^{2} \xi_{1}^{2}-54 \xi_{2} \xi_{1} \xi_{3}+81 \xi_{3}^{2}+12 \xi_{3} \xi_{1}^{3}}\right]^{(1 / 3)}}-\frac{1}{3} \xi_{1} \\
& +\frac{1}{2} \mathrm{i} \sqrt{3}\left\{\frac{1}{6}\left[36 \xi_{2} \xi_{1}-108 \xi_{3}-8 \xi_{1}^{3}+12 \sqrt{12 \xi_{2}^{3}-3 \xi_{2}^{2} \xi_{1}^{2}-54 \xi_{2} \xi_{1} \xi_{3}+81 \xi_{3}^{2}+12 \xi_{3} \xi_{1}^{3}}\right]^{(1 / 3)}\right. \\
& \left.+\frac{6\left(\frac{1}{3} \xi_{2}-\frac{1}{9} \xi_{1}^{2}\right)}{\left[36 \xi_{2} \xi_{1}-108 \xi_{3}-8 \xi_{1}^{3}+12 \sqrt{12 \xi_{2}^{3}-3 \xi_{2}^{2} \xi_{1}^{2}-54 \xi_{2} \xi_{1} \xi_{3}+81 \xi_{3}^{2}+12 \xi_{3} \xi_{1}^{3}}\right]^{(1 / 3)}}\right\} \\
& q_{2}=-\frac{1}{12}\left[36 \xi_{2} \xi_{1}-108 \xi_{3}-8 \xi_{1}^{3}+12 \sqrt{12 \xi_{2}^{3}-3 \xi_{2}^{2} \xi_{1}^{2}-54 \xi_{2} \xi_{1} \xi_{3}+81 \xi_{3}^{2}+12 \xi_{3} \xi_{1}^{3}}\right]^{(1 / 3)} \\
& +\frac{3\left(\frac{1}{3} \xi_{2}-\frac{1}{9} \xi_{1}^{2}\right)}{\left[36 \xi_{2} \xi_{1}-108 \xi_{3}-8 \xi_{1}^{3}+12 \sqrt{12 \xi_{2}^{3}-3 \xi_{2}^{2} \xi_{1}^{2}-54 \xi_{2} \xi_{1} \xi_{3}+81 \xi_{3}^{2}+12 \xi_{3} \xi_{1}^{3}}\right]^{(1 / 3)}}-\frac{1}{3} \xi_{1} \\
& -\frac{1}{2} \mathrm{i} \sqrt{3}\left\{\frac{1}{6}\left[36 \xi_{2} \xi_{1}-108 \xi_{3}-8 \xi_{1}^{3}+12 \sqrt{12 \xi_{2}^{3}-3 \xi_{2}^{2} \xi_{1}^{2}-54 \xi_{2} \xi_{1} \xi_{3}+81 \xi_{3}^{2}+12 \xi_{3} \xi_{1}^{3}}\right]^{(1 / 3)}\right. \\
& \left.+\frac{6\left(\frac{1}{3} \xi_{2}-\frac{1}{9} \xi_{1}^{2}\right)}{\left[36 \xi_{2} \xi_{1}-108 \xi_{3}-8 \xi_{1}^{3}+12 \sqrt{12 \xi_{2}^{3}-3 \xi_{2}^{2} \xi_{1}^{2}-54 \xi_{2} \xi_{1} \xi_{3}+81 \xi_{3}^{2}+12 \xi_{3} \xi_{1}^{3}}\right]^{(1 / 3)}}\right\}
\end{aligned}
$$


ان التوصل الى حالة الاستقرار في نظام معادلات المياه الضحلة يتأثر بدرجة كبيرة بالكميات

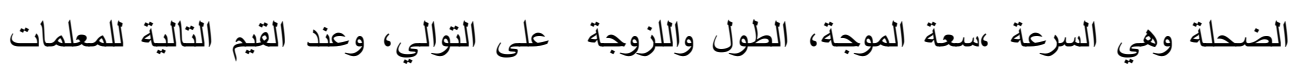

$$
\begin{aligned}
& g=9.8 \quad \mathrm{bu}_{2}=1 \quad \mathrm{bv}_{2}=1 \quad \Omega=\frac{\pi}{12} \quad \psi=0.5 \quad \mathrm{fu}_{2}=0.131 \quad \mathrm{fv}_{2}=0.131 \\
& f u=\Omega \sin (\Psi)=0.131 \quad \mathrm{k} 1=2 \quad \mathrm{k} 2=2 \quad \mathrm{u}_{0}=1.3 \quad \mathrm{~L}=-1 \quad \mathrm{\eta}_{0}=0.5 \\
& \text { وبتعويضها بالمعادلة ( 23) نحصل على : } \\
& \lambda^{3}+(-3.88 \alpha+2.3) \lambda^{2}+\left(4.5461 \alpha^{2}-4.647 \alpha-18.27099791\right) \lambda+\left(-1.42805 \alpha^{3}+\right. \\
& \left.1.9435 \alpha^{2}+32.45949 \alpha-12.740\right)=0 \\
& \text { ومن النتائج العددية، تبرز الحالات الآتية التي تميز حالة (الاستقرار من عدم الاستقرار) لنظام }
\end{aligned}
$$

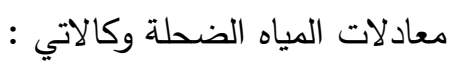

$$
\begin{aligned}
& \text { أولا:- الحالة الأولى: يكون نظام معادلات المياه الضحلة مستقراً في الحالات الآتية : } \\
& b_{u_{2}}=b_{v_{2}} \quad \text { و } \\
& \alpha \leq-3.6 \quad, \quad 1.2 \leq \alpha \leq 3 \quad-2 \\
& L<0 \quad, \quad 0.1 \leq L \leq 1.9 \quad, \quad 9 \leq L \leq 10 \quad-3 \\
& 0.9 \leq u_{0} \leq 1.8 \quad-4
\end{aligned}
$$

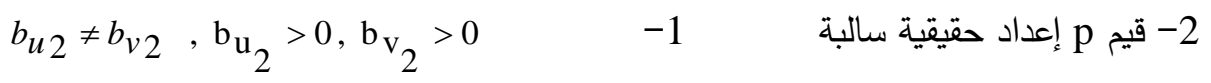

$$
\begin{aligned}
& L=0 \quad-2 \\
& u_{0}<0 \quad u_{0} \geq 1.9 \quad-3
\end{aligned}
$$

ثانيا : - الحالة الثانية: يكون نظام معادلات المياه الضحلة غير مستقر في الحالات آلاتية :

$$
\begin{aligned}
& b_{u_{2}}=b_{v_{2}}, \quad b_{u_{2}} \leq-2, \mathrm{~b}_{\mathrm{v}_{2}} \leq-2 \quad-1 \quad \text { - } \\
& \alpha \geq 3.1 \quad-2 \\
& L \geq 11 \quad-3 \\
& \text { - } b_{u_{2}} \neq b_{v_{2}}, b_{u_{2}}<0, b_{v_{2}}<0 \text { قيم أعداد حقيقية موجبة عند p }
\end{aligned}
$$




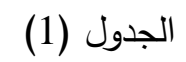

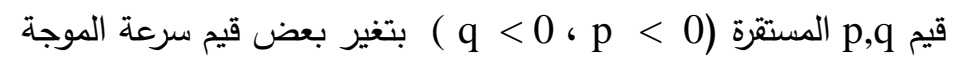

\begin{tabular}{|l|l|l|}
\hline$\alpha$ & \multicolumn{1}{|c|}{$q_{1}, q_{2}$} & \multicolumn{1}{c|}{$\mathrm{p}$} \\
\hline-3.6 & $-1.669110413 \pm 380424589 \mathrm{i}$ & -12.9297717 \\
\hline-3.7 & $-1.77662643 \pm 3.382926108 \mathrm{i}$ & -13.10265471 \\
\hline-3.8 & $-1.884502042 \pm 3.383552027 \mathrm{i}$ & -13.27499592 \\
\hline-3.9 & $-1.992590380 \pm 3.382315973 \mathrm{i}$ & -13.44681924 \\
\hline-4 & $-2.100929873 \pm 3.379227782 \mathrm{i}$ & -13.61814025 \\
\hline-4.1 & $-2.209513149 \pm 3.374293605 \mathrm{i}$ & -13.78897370 \\
\hline-4.2 & $-2.318333222 \pm 3.36751595 \mathrm{i}$ & -13.95933356 \\
\hline-4.3 & $-2.427383465 \pm 358893739 \mathrm{i}$ & -14.12923307 \\
\hline-4.4 & $-2.536657593 \pm 3.348422248 \mathrm{i}$ & -14.29868481 \\
\hline-4.5 & $-2.646149632 \pm 3.336093115 \mathrm{i}$ & -14.46770074 \\
\hline-4.6 & $-2.755853905 \pm 3.321894245 \mathrm{i}$ & -14.63629219 \\
\hline$\cdot$ & $\cdot$ & $\cdot$ \\
\hline & $\cdot$ &. \\
\hline & $\cdot$ &. \\
\hline-100 & $-171.2477660 \pm 14.45043007 \mathrm{i}$ & -47.80446809 \\
\hline
\end{tabular}

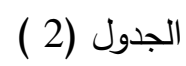

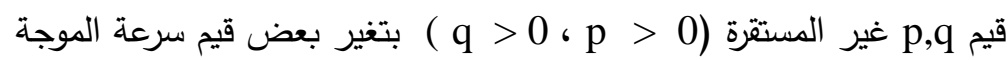

\begin{tabular}{|c|c|c|}
\hline$\alpha$ & $q_{1}, q_{2}$ & $\mathrm{p}$ \\
\hline 10 & $16.6087165 \pm 2.231835409 \mathrm{i}$ & 3.282566973 \\
\hline 11 & $18.23662331 \pm 2.371432634 \mathrm{i}$ & 3.906753387 \\
\hline 12 & $19.87287394 \pm 2.499991373 \mathrm{i}$ & 4.514252118 \\
\hline 13 & $21.51600871 \pm 2.619823613 \mathrm{i}$ & 5.107982590 \\
\hline 14 & $23.16486497 \pm 2.732573465 \mathrm{i}$ & 5.690270067 \\
\hline 15 & $24.81851161 \pm 2.839447050 \mathrm{i}$ & 6.262976789 \\
\hline 16 & $26.47619815 \pm 2.941352835 \mathrm{i}$ & 6.827603706 \\
\hline 17 & $28.13731558 \pm 3.03891033 \mathrm{i}$ & 7.385368840 \\
\hline 18 & $29.80136623 \pm 3.13292230 \mathrm{i}$ & 7.937267546 \\
\hline 19 & $31.46794055 \pm 3.223557253 \mathrm{i}$ & 8.484118893 \\
\hline 20 & $33.13669925 \pm 3.311284731 \mathrm{i}$ & 9.026601497 \\
\hline$\cdot$ & $\cdot$ &. \\
$\cdot \cdot$ & $\cdot$ &. \\
$\cdot$. & $\cdot$ &. \\
\hline 100 & $168.0027048 \pm 7.447749846 \mathrm{i}$ & 49.69459040 \\
\hline
\end{tabular}




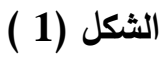

قيم p التي يكون عندها نظام معادلات المياه الضحلة غير مستقر

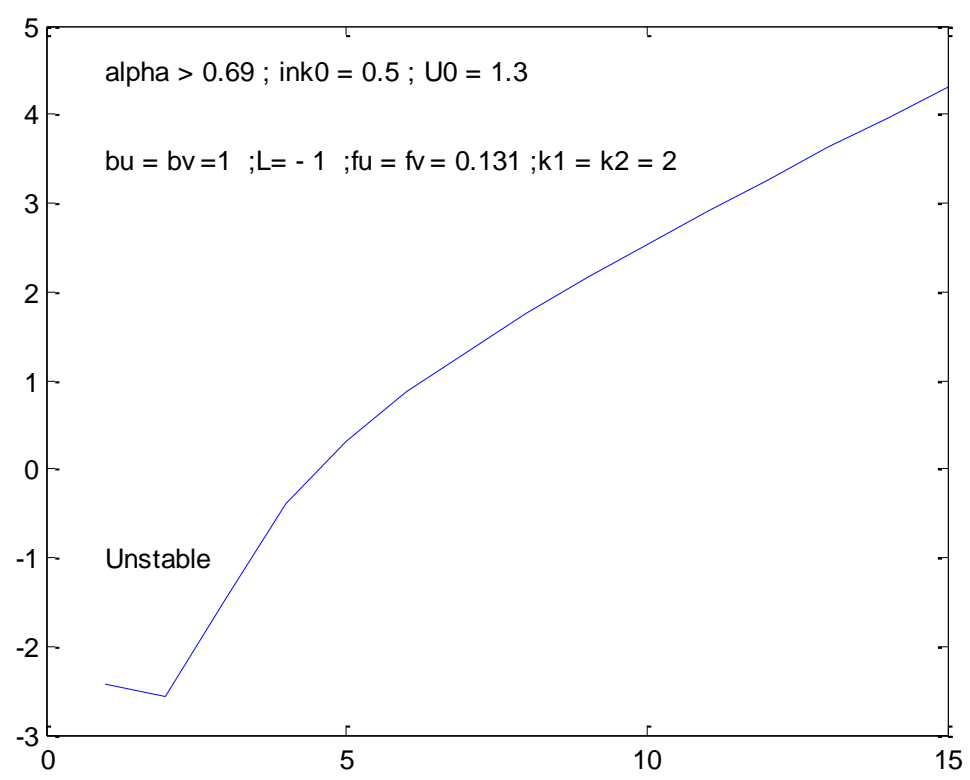

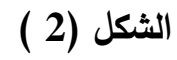

قيم p التي يكون عندها نظام معادلات المياه الضحلة مستقراً

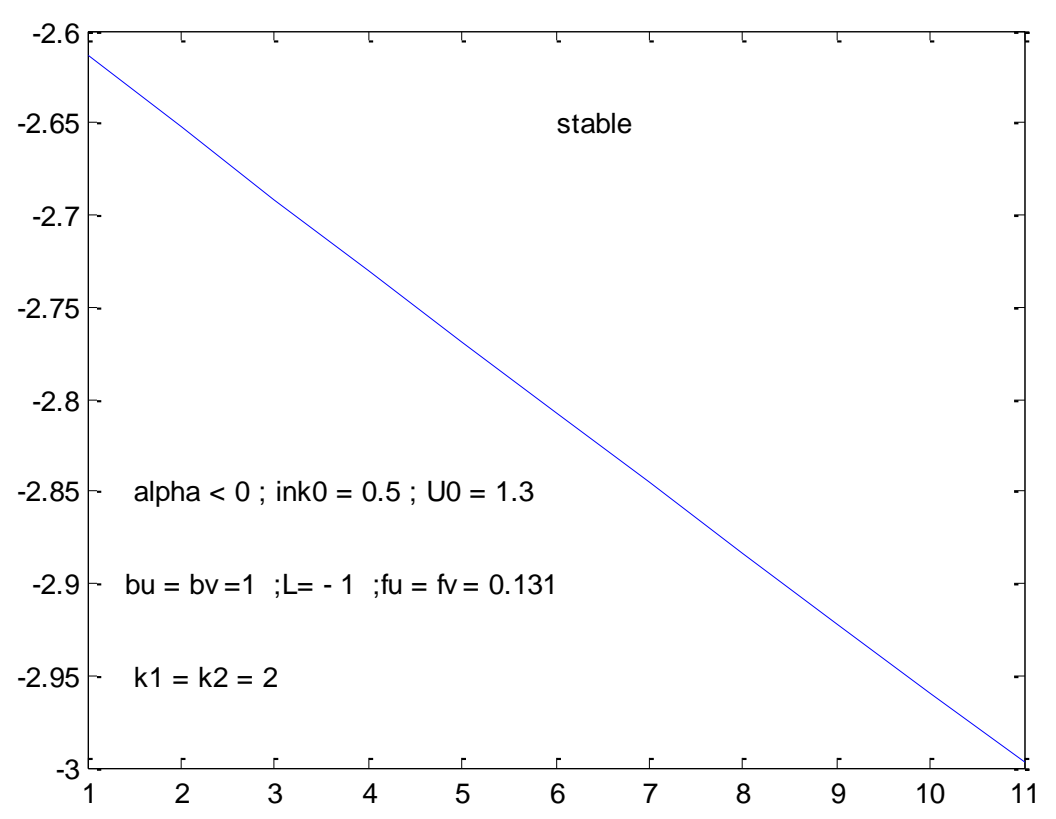




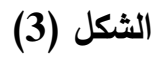

قيم q التي يكون عندها نظام معادلات المياه الضحلة غير مستقر

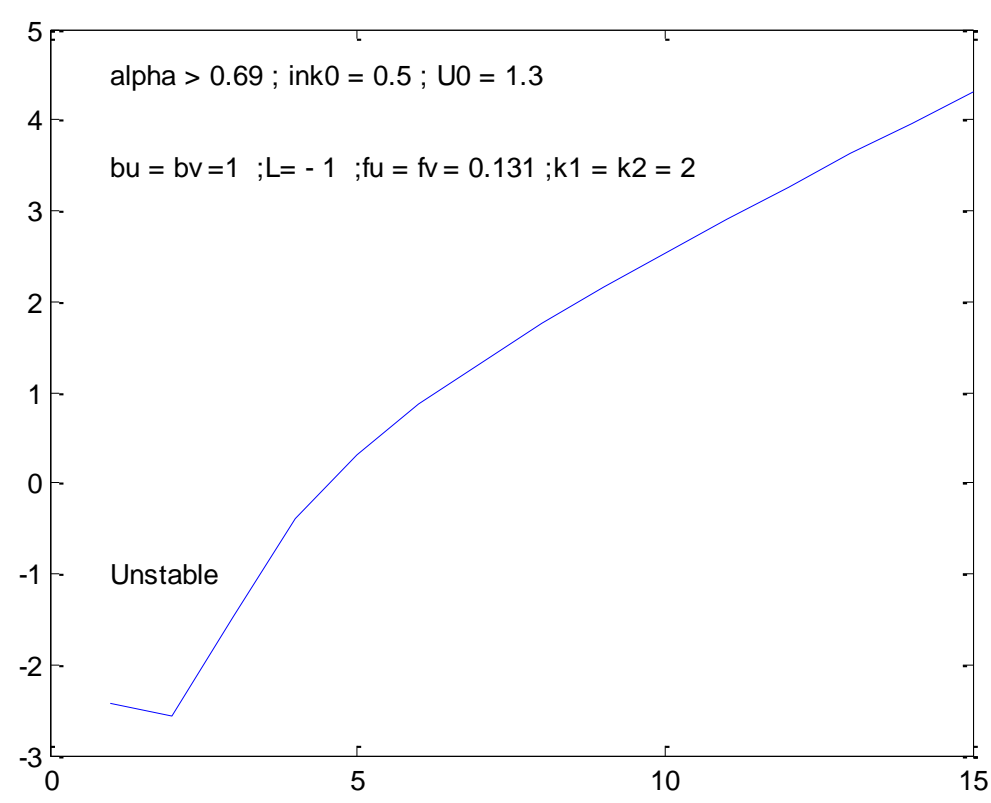

(4) الثكل

قيم q التي يكون عندها نظام معادلات المياه الضحلة مستقراً

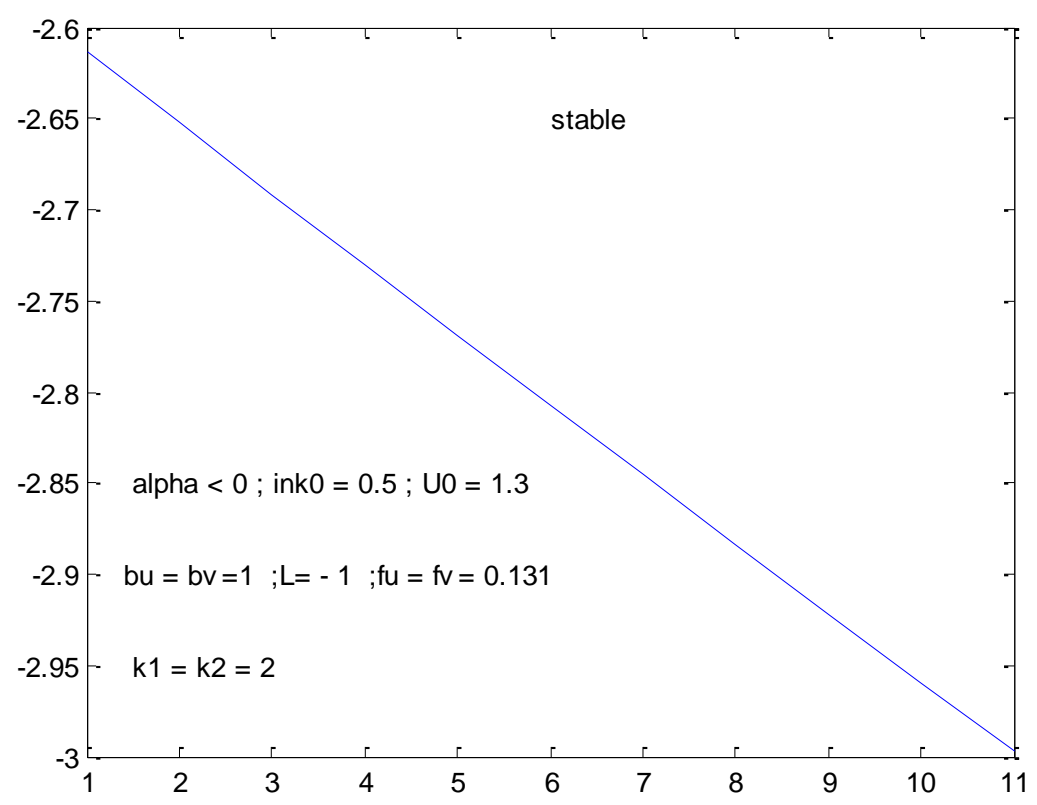




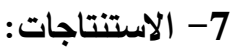

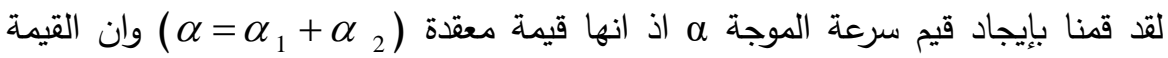
الموجبة او السالبة لـ $\alpha_{1}$ تؤثر في حالة نمو الاضطراب او تلاشيه. لقد تمت تجزئة نظام

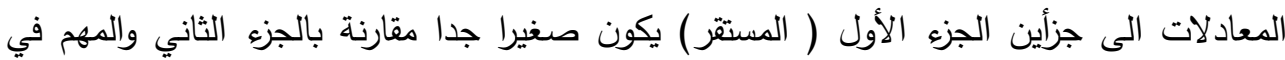
تحليل الاستقرارية، وتم إيجاد الحل، القيم الذاتية ( EigenValues ) والمعادلة المميزة بالنسبة للجز الثاني،ثم قمنا بحلها برمجيا باستخدام نظام (Characteristic polynomial)

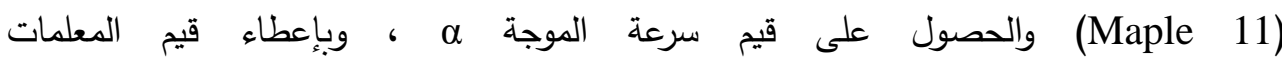
تبين ان هذه المنظومة تكون في ( $g$, bu $\left.2, b v, \Omega, \psi, f_{2}, f v_{2}, k 1, k 2, u_{0}, L, \eta_{0}\right)$ حالة استقرار عند : 1 - قيم q إعداد مركبة

$$
\begin{aligned}
& b_{u_{2}}=b_{v_{2}} \quad g \quad \mathrm{~b}_{\mathrm{u}_{2}} \geq 0, \mathrm{~b}_{\mathrm{v}_{2}} \geq 0 \quad-1 \\
& \alpha \leq-3.6 \quad, \quad 1.2 \leq \alpha \leq 3 \quad-2 \\
& L<0 \quad, \quad 0.1 \leq L \leq 1.9 \quad, \quad 9 \leq L \leq 10 \quad-3 \\
& 0.9 \leq u_{0} \leq 1.8 \quad-4 \\
& b_{u_{2}} \neq b_{v_{2}}, \mathrm{~b}_{\mathrm{u}}>0, \mathrm{~b}_{\mathrm{v}_{2}}>0 \quad-1 \quad \text { قيم } \\
& L=0 \quad-2 \\
& u_{0}<0 \quad g u_{0} \geq 1.9 \quad-3
\end{aligned}
$$

وبعكسه فان المنظومة تكون في حالة عدم استقرار. 


\section{|لمصادر}

[1] Ahmad, R. A., 2003, " A Mathematical Model for the Effect of Electrical Conductivities of the Wall on the stability of fluid flow and Transition to chaos"chapter(4), pp35-43.

[2] AL-Obaidi, M.F. and Abraham , B.M., 2001"Stability Analysis and Chaos in a Bend Duct”, Raf. J. Sci., Vol. 12, No. 1, pp. 91-99.

[3] Antonio, D.Delgado and Tomas C.Rebollo, 2004 "Asymptotically balnced schemes for non-homogeneous hyperbolic systems application to the Shallow Water equations"journal of Comptes Rendus math., ISSN 1631- 073x, pp85-90 .

[4] Bohorquez and Fernandez-Feria,2006 " Non parallel spatial stability of shallow water flow down an inclined plane of arbitrary slop".

[5] Carlos, C.Pena and Luis P.Thomas,2006, "Soluitions of shallow water equations In Non-rectangular Cross- Section channels".

[6] David, A.Randall, 2006 ,"The Shallow Water Equations”,pp1-3.

[7] David, L. George, 2004 " Numerical Approximation of the Nonlinear Shallow Water Equations with Topography and Dry Beds",pp5-8.

[8] Fortunato, M., Kurizki, G. and Schleich W.P., 1998 “ Stabilization of Deterministically Chaotic Systems by Interference and Quantum Measurements The Ikeda Map Case Physical Review Letters", Vol. 80, No.26,PP 5730-5733.

[9] Gilbert, S. , 1980, “Linear Algebra and it's Applications",second Edition, Academic Press, New York .

[10] Henry, M. P. and Robert J.S., 1978 "Introduction to Dynamics and control”, McGraw-Hill,London .

[11] Logan, J. D., 1987 “Applied Mathematics Wiley and Sony”.

[12 ] Mark, A.Taylor and William F.Spotz,1999, "Fast and Hight - order solution to the Sperical shallow watr equation"

[13] Monagan, M.B., 2003, “ Maple 11 Advanced Programming Guid ”.

[14] Thomas, L.Harman, 2000, “Advanced Engineering Mathematics with Matlab",second edition. 
[15] Shallow Water equations -Wikipedia,2007, http://en.wikipedia.org/wiki/Shallow water equations.

[16] Yong, Zhou,2000, "Infinite propagation speed for ashallow water equation".

[17] Zhaug, D. S ., Wei G. W., Kouri D. J. \& Hoffman Q.K., 1997, "Burger's Equation with High Reynolds Number", J. Phys. Fluid 9(6), pp. $1853-1855$. 